\title{
The effects of diet and vanadyl sulfate supplementation on blood glucose levels of diabetics: review of current human data and recommendations for further study
}

\begin{abstract}
Studies in the literature have shown that vanadium supplementation has an insulin mimetic effect on the blood glucose levels of both animals and humans. ${ }^{1-8}$ In the current literature, several studies have examined the effects of vanadium supplementation on blood glucose levels of both type 1 and type 2 diabetics. However, dietary intake of vanadium has not been considered. It is highly probable that vanadium from food sources may be more effective than supplementation alone due to the synergistic effects of the various food components. This raises several questions: Could a diet high in food sources of vanadium be more effective in controlling blood sugar levels in diabetics compared with vanadium supplements alone? Could a diet high in food sources of vanadium increase the intestinal absorption of vanadium? Could a diet high in food sources of vanadium reduce the need for vanadium supplements while maintaining the effects of lowering blood glucose levels in diabetics. ${ }^{5,8}$ This review examines current human studies in the literature which report the lowering effects of vanadium on blood glucose levels of both type 1 and type 2 diabetics. The authors also recommend further study to determine whether a diet high in vanadium containing foods will improve blood glucose levels in type 1 and type 2 diabetics thereby providing an alternative and complementary means of managing both type 1 and type 2 diabetes.
\end{abstract}

Volume 2 Issue 3 - 2015

\author{
Lorna C Shepherd, Hannah Lima and Mary \\ Ott \\ Department of Nutrition, Life University, USA
}

Correspondence: Lorna C Shepherd, Associate Professor, Department of Nutrition, Life University, 1269 Barclay Circle, Marietta, GA 30060, USA, Tel 7704262742,

Email lorna.shepherd@life.edu

Received: March 19, 2015 | Published: June 03, 2015

Keywords: vanadyl sulfate, vanadium, supplementation

Abbreviations: FBG, fasting blood glucose; HgbA1C, hemoglobin A1C;mcg, micrograms; mg/day, milligrams per day; DEXA, x-ray absorptiometry; CMP, comprehensive metabolic panel; ANOVA, analysis of variance

\section{Introduction}

A number of studies have suggested the ability of vanadium to improve the blood glucose control of diabetics and also to improve the negative side effects associated with diabetes. Human studies have specifically shown a reduction in blood glucose levels and hemoglobin A1C (hgbA1C) levels in both type 1 and type 2 diabetics. ${ }^{2,3,5,8}$ Goldfine et al., ${ }^{5}$ reported improved insulin sensitivity in type 2 diabetics who were given vanadium supplements and Soveid et al., ${ }^{8}$ indicated that type 1 diabetics, who were supplementing with vanadium, showed a decreased need for insulin. Vanadium is found in many food sources. Vanadium is found abundantly in food items such as dill seeds which contain $430 \mathrm{mcg} / \mathrm{kg}$ and black pepper which contains $987 \mathrm{mcg} / \mathrm{kg}$. Foods such as whole grains, seafood, meats and dairy provide $5-30 \mathrm{mcg}$ of vanadium per kilogram and smaller amounts of vanadium, $1-5 \mathrm{mcg} / \mathrm{kg}$, can be obtained from oils, fats and vegetables. According to Ulbricht et al., ${ }^{9}$ a safe level of vanadium intake for humans is less than $1.8 \mathrm{mg}$ per day. However, the average diet provides about $6-20 \mathrm{mcg}$ of vanadium per day. ${ }^{9}$

Mishra et al., ${ }^{10}$ reported that vanadium can activate the tyrosine kinase and glycogen synthase activity of insulin receptors in adipocytes. ${ }^{10}$ Vanadium can also increase the concentration of fructose 2, 6-biphosphate which can activate glycolysis in hepatocytes. Vanadium salts mimic several of the effects of insulin which include metabolic and growth promoting factors, and have also been shown to stimulate glycogen synthesis in muscles. ${ }^{10}$

\section{Vanadyl sulfate}

Vanadium as vanadyl sulfate is believed to be a potent regulator of fasting blood glucose by improving the sensitivity to insulin at the receptor level. Vanadyl sulfate is known to be 6-10times less toxic than other forms of vanadium such as vanadate. ${ }^{11}$ In fact, ten percent of dietary vanadium exists as vanadyl sulfate. ${ }^{12}$ Based on an analysis of the current literature, vanadyl sulfate shows very promising results with regard to management of diabetes. Vanadyl sulfate has been studied more extensively in both rat and human studies and produced more significant results with less risks of toxicity. Several human studies were reviewed in which both type 1 and type 2 diabetics were participants. Soveid et al., ${ }^{8}$ conducted a study in which they examined the effects of vanadyl sulfate supplementation on the blood glucose levels of type 1 diabetics. This study showed very promising results. The study was conducted for a period of 30months. During weeks 2-5, study participants were given an adaptive dose of $40-60 \mathrm{mg} /$ day of vanadyl sulfate. The dosage was then progressively increased to 225 $300 \mathrm{mg} /$ day which was given in three equal dosages for the duration of the study. ${ }^{8}$ Findings from this most recent study revealed that insulin requirements decreased thirty percent (30\%) among participants from 
37.2 to 25.8 units per day. Fasting blood glucose levels among study participants also decreased from 250 to $152 \mathrm{mg} / \mathrm{dl}$, whereas afternoon blood glucose levels showed a significant decrease from $250 \mathrm{mg} / \mathrm{dl}$ to $186 \mathrm{mg} / \mathrm{dl}$. The insulin requirements of two participants were reduced to less than 10 units per day and thirty-five percent (35\%) of study participants were able to reduce their daily injections of insulin to only once per day. Soveid et al., ${ }^{8}$ reported that the results occurred primarily during the first three months of the study but remained stable throughout the duration of the study. These results were also consistent after one month post discontinuation of the vanadyl sulfate supplementation. ${ }^{8}$

The findings from a study conducted by Goldfine et al., ${ }^{5}$ provided evidence about the importance of dosage as a factor in efficacy. The Goldfine ${ }^{5}$ study examined the effects of vanadyl sulfate supplementation in a group of type 2 diabetics. Dosages of vanadyl sulfate ranging from $75 \mathrm{mg}$ /day to $300 \mathrm{mg}$ /day were administered three (3)times per day in smaller doses. The duration of this study was 12 weeks and placebos were administered prior to the vanadyl sulfate regimen and subsequent to the discontinuation of the supplementation. The results of the study showed a significant decrease in the hemoglobin A1C (hgbA1C) levels, from 7.8 to 6.8 , in the group supplemented with
$150 \mathrm{mg}$ of vanadyl sulfate per day. A decrease in hgbA1C levels from 7.1 to 6.8 was also observed in the group supplemented with $300 \mathrm{mg} /$ day of vanadyl sulfate. There was also a significant decrease in fasting blood glucose levels from 167.2 to 144.1 in the group supplemented with $300 \mathrm{mg}$ of vanadyl sulfate per day. Findings from this study also indicated that, at a $95 \%$ compliance rate, there were no side effects reported with a $75 \mathrm{mg} /$ day dosage of vanadyl sulfate, whereas, there were some complaints of gastrointestinal distress at a dosage of $150 \mathrm{mg} /$ day of vanadyl sulfate. All participants who were given the $300 \mathrm{mg} /$ day dosage reported experiencing cramping, abdominal discomfort and/or diarrhea. However, there was no evidence of biochemical toxicities at any dosage level or any reports of hypoglycemia which required attention for any of the participants. ${ }^{5}$

Of all the forms of vanadium, the reviewed studies revealed that vanadyl sulfate was the most effective at doses ranging from $100 \mathrm{mg} /$ day to $300 \mathrm{mg} /$ day and when taken in three equal daily doses. Although some gastrointestinal discomfort was reported, these side effects could be minimized or alleviated by starting with a lower transitional or adaptation dose and gradually increasing the dosage throughout the duration of the study (Table 1).

Table I Pre and post FBG and AIC levels in human studies

\begin{tabular}{lllll}
\hline & Pre-FBG & Post-FBG & Pre-AlC & Post-AIC \\
\hline Cohen et al. $^{3}$ & $210+19$ & $181+14$ & $9.7+0.5 \%$ & $8.8+0.6 \%$ \\
Goldfine et al. $^{5}$ & $167.2+72.9$ & $144.1+66.8$ & $10.3+2.6 \%$ & $7.1 / 7.8+1.7 / 2.3 \%$ \\
Afkhami-Ardekani et al. $^{\prime}$ & $175.5+35.2$ & $161.6+36.9$ & $7.8+0.9 \%$ & $7.8+0.8 \%$ \\
Boden et al. $^{2}$ & $167.57+32.43$ & $133.33+25.22$ & Not given & Not given \\
Soveid et al. $^{8}$ & $238+71$ & $152+42$ & Not done & Not done \\
\hline
\end{tabular}

\section{Conclusion}

Based on the current studies available and reviewed, supplementation with $300 \mathrm{mg}$ per day of vanadyl sulfate had the greatest and most significant impact on fasting blood glucose levels of diabetics. In addition, the studies that were reviewed indicated that fasting blood glucose levels remained stable for up to one month after cessation of the study regimen. Findings also suggested that another potential advantage of vanadyl sulfate supplementation is its ability to decrease total cholesterol levels. ${ }^{2,3,5,8}$ Human studies reviewed did not examine the effects of dietary intake of vanadium. It is highly probable that studies could show beneficial effects with lower doses of vanadyl sulfate supplementation. These effects are most likely to be evident in those studies that focus on dietary intake of vanadium rich food sources as well as on supplementation of vanadium. Only one study was designed to examine the long term benefits of vanadyl sulfate supplementation and was of 30 months duration. Other studies were of shorter duration with the study regimen generally averaging between 1-3months in length. Further studies are definitely needed to determine the efficacy and safety of vanadyl supplementation for long term management of type 1 and type 2 diabetes. Future studies may also be designed to determine the potential benefits of intermittent supplementation.

\section{Future directives}

Based on a review of the literature, vanadyl sulfate supplementation has never been studied in conjunction with diet. The authors propose a preliminary study to look at the synergistic effects of a diet high in food sources of vanadium in addition to vanadyl sulfate supplementation. The proposed study will help to determine whether a diet high in vanadium containing foods will further improve blood glucose levels in diabetics who are taking vanadium supplements. The authors hypothesize that a diet high in food sources of vanadium, along with vanadyl sulfate supplementation, will further improve blood glucose levels in diabetics compared to a diet low in food sources of vanadium and/or vanadyl sulfate supplements alone. Study participants will be recruited from the population of metro Atlanta. Flyers will be placed in doctor's offices, churches, colleges, and community centers. In addition, an online craigslist advertisement will be posted. Based on the methodology utilized in the vanadium supplementation studies referenced above and assessment, a total of thirty (30) type one and type two diabetics will be selected. Exclusion criteria for potential study participants are the history or occurrence of hepatic issues, renal issues, cardiovascular disease, respiratory issues, anemia, bleeding disorders, abnormal hemoglobin/hematocrit, or unstable weight in the last two months prior to recruitment. Pre-menopausal women will also be excluded unless there is proof of current contraception or sterility.

The study is of three (3) months duration and is designed so that study participants are randomly divided into three equal groups; a control group in which participants receive a placebo and stay on their regular diets, one group in which participants receive vanadyl sulfate supplementation along with a diet low in food sources of vanadium, and the third group of participants receive vanadyl sulfate supplementation along with a diet rich in food sources of vanadium. The vanadyl sulfate supplementation groups will begin supplementation with an adaptation dose of $100 \mathrm{mg} /$ day of vanadyl sulfate per day which will 
be gradually increased to $150 \mathrm{mg}$ /day in subsequent weeks. During weeks nine and ten, vanadyl sulfate supplementation will cease but participants in the supplement groups will continue to follow their diets which are high or low in vanadium food sources. Participants will be provided with food lists which indicate the allowable foods for their assigned diet groups. Dietary adherence will be determined via weekly twenty-four (24) hour food recalls and three (3) day food logs will be collected weeks $2,4,6$ and 8 . The study participants will selfmonitor blood glucose at pre-determined times throughout each week. Logs of three fasting blood glucose levels and three pre/post prandial blood glucose readings will be collected each week. Both blood glucose monitors and test strips will be provided to the participants. HgbA1C, Dual Energy X-ray Absorptiometry (DEXA) scan and a Comprehensive Metabolic Panel (CMP) will be obtained at baseline and at the end of the ten (10) week period. Analysis of Variance (ANOVA) will be utilized to assess the differences among the means for the three comparison groups. It is expected that findings from the proposed study will add to the current body of knowledge regarding insulin mimetic effects of the trace mineral vanadium. This proposed study which investigates the effects of dietary sources of vanadium, incorporated into the diet of type 1 and type 2 diabetics, along with vanadyl sulfate supplementation has the potential to benefit society by providing evidence of an alternative and complementary means of managing both type 1 and type 2 diabetes.

\section{Acknowledgements}

None.

\section{Conflict of interest}

The author declares no conflict of interest.

\section{References}

1. Afkhami-Ardekani M, Karimi M, Mohammadi SM, et al. Comparison of the Effects of Sodium metavanadate and Zinc Sulfate Supplementation on Lipid and Glucose in Patients with Type 2 Diabetes. Iranian Journal of Diabetes and Obesity. 2009;1(1):22-29.
2. Boden G, Chen X, Ruiz J, et al. Effects of vanadyl sulfate on Carbohydrate and lipid metabolism in patients with non-insulindependent diabetes mellitus. Metabolism. 1996;45(9):1130-1135.

3. Cohen N, Halberstam M, Shlimovich P, et al. Oral vanadyl sulfate improves hepatic and peripheral insulin sensitivity in patients with noninsulin-dependent diabetes mellitus. J Clin Invest. 1995;95(6):25012509 .

4. García-Vicente S, Yraola F, Marti L, et al. Oral insulin-mimetic compounds that act independently of insulin. Diabetes. 2007;56(2):486493.

5. Goldfine AB, Patti ME, Zuberi L, t al. Metabolic effects of vanadyl sulfate in humans with non-insulin-dependent diabetes mellitus: In vivo and in vitro studies. Metabolism. 2000;49(3):400-410.

6. Kim JM, Chung JY, Lee SY, et al. Hypoglycemic effects of vanadium on alloxan monohydrate induced diabetic dogs. J Vet Sci. 2006;7(4):391395.

7. Nechay BR. Mechanisms of action of vanadium. Annu Rev Pharmacol Toxicol. 1984;24:501-524.

8. Soveid M, Dehghani GA, Omrani GR. Long- term efficacy and safety of vanadium in the treatment of type 1 diabetes. Arch Iran Med. 2013;16(7):408-411.

9. Ulbricht C, Chao W, Costa D, et al. An evidence-based systematic review of vanadium by the natural standard research collaboration. $J$ Diet Suppl. 2012;9(3):223-251.

10. Mishra SK, Kumar A, Chaturvedi RK, et al. Vanadium salts versus diabetes: An overview. Syst Rev Pharm. 2010;1(2):172-174.

11. Vanadium/Vanadyl Sulfate. Altern Med Rev. 2009;14(2):177-180.

12. Vanadic sulfate. Pub Chem Open Chemistry Database. 2004. 